\title{
EVOLUCIÓN DEL CONOCIMIENTO FÍSICO
}

\author{
GARCÍA BARNETO, A. y BARRIOS ARIAS, V. \\ CEP de Huelva. Mora Claros, 1. 21001 Huelva.
}

\begin{abstract}
SUMMARY
In this paper we wish to present the evolution of the structure of the physic knowledge from the level of 1 st year BUP to science graduates. The reliability of the responses also analysed.
\end{abstract}

\section{INTRODUCCIÓN}

Con frecuencia, los conocimientos científico y ordinario son diferenciados únicamente por el volumen de hechos $y$ conceptos que abarcan. Sin embargo, la investigación didáctica ha mostrado que existen otros aspectos relacionados con la estructuración de las informaciones, que caracterizan mejor las diferencias entre expertos y novatos de una disciplina científica (Pozo 1987).

En este artículo tratamos de demostrar que la organización de la información en redes complejas es un factor que distingue a ambos grupos de una manera más precisa, de forma que una atención centrada en cuantificar el conocimiento puntual de algún aspecto concreto de la disciplina científica, como en muchas ocasiones se hace, puede llegar a desorientar sobre todo si está relacionado con preconcepciones muy arraigadas. Para ello, y sirviéndonos del análisis de representaciones, vamos a esquematizar la estructura del conocimiento científico por medio de un parámetro que sea capaz de medir ei conocimiento simultáneo y significativo (Ausubel, et al. 1983 ) de conceptos físicos. Su variación mientras dura la formación científica nos revelará como se modifica la trama de relaciones establecidas entre los conceptos que se han ido conociendo.

El análisis de las ideas alternativas habituales (Hierrezuelo y Montero 1989) es un instrumento versátil que, por ejemplo, Gagliardi (1988) emplea para determinar los obstáculos epistemológicos de los alumnos. En esta línea, dicha técnica nos pareció útil para aclarar hasta qué punto el contacto con información científica, en aulas de institutos y universidad, es eficaz en el proceso de asimilación de la compleja estructura de las disciplinas tradicionales. La amplitud de la tarea obligaba a acotar el número de campos de conocimiento a investigar, por ello nos centramos en mecánica, ámbito bien estudiado y en el que nos podríamos mover con referentes de garantia, y dentro de ella en las siguientes cuestiones: rozamiento, movimiento de partículas en gases, fuerza, caída y energía.

El resultado de la investigación no deja dudas acerca de dos cosas: de la ingente tarea que nos queda a los profesores para lograr que nuestros alumnos construyan un cuerpo coherente de conocimientos (similar al que manejan los físicos o plantean los libros de texto), y de lo ilusoria que es la creencia de muchos profesores que plantean la enseñanza de la ciencia como la transmisión de una materia acabada, construida por completo, con explicaciones para todo, sin elementos de duda ni im. plicaciones sociales, y que suponen conclusa no sólo al final del bachillerato o la universidad, sino al término de cualquiera de sus cursos.

\section{INVESTIGACIÓN DISEÑO Y CARACTE- RIS'TICAS}

Como se ha indicado, el objetivo de nuestra investigación ha sido conocer la evolución de las ideas previas sobre algunas cuestiones físicas concretas a lo largo del 
bachillerato y la universidad. Además, una vez aclarado esto, era también intención nuestra analizar la simultaneidad con que se dan los conocimientos significativos en las cuestiones analizadas, de forma que pudiera apreciarse la estructura conceptual que el alumno tiene de la mecánica después de recibir la información que en los centros se ha estimado adecuada. Contemplar cómo evoluciona aquélla con el tiempo puede ser de lo más clarificador en orden a extraer consecuencias para la enseñanza (Driver 1988, Giordan 1989).

En la primera fase de la investigación, para conocer las representaciones de los alumnos, hemos empleado una encuesta con ítems de respuesta múltiple (Anexo I). En cada uno de ellos se plantean cuatro posibilidades además de citar explícitamente las de «no lo sé» y «otra respuesta». Además, junto a una explicación abierta de la opción escogida, siempre se demanda una indicación del nivel de seguridad en la respuesta por medio de una escala del 0 (seguridad nula) al 10 (seguridad completa). En el caso del rozamiento la pregunta es completamente abierta, invitando al encuestado a que reconozca y explique el origen de las fuerzas que actúan sobre un vehículo de motor que circula por un tramo horizontal de carretera. Ello nos ha permitido clasificar las respuestas en cuatro niveles de conocimiento acerca del concepto de rozamiento, desde los que ni siquiera lo usan (A) a los que entienden su origen, efectos y mecanismos de acción (D), pasando por los que lo reducen a la fricción entre sólidos que deslizan (B) y los que entienden significativa la fricción fluida (C). Se plantean dos ítems en referencia a cada temática.

La muestra ha sido formada por 250 alumnos de tres institutos de Huelva que recibŕan una educación científica típica, homologable a la de la mayoría de centros españoles, y por 31 graduados de ciencias que impartían docencia o se preparaban para ello. La encuesta fue pasada al final de curso.

\section{RESULTADOS Y DISCUSIÓN}

Basándonos en nuestra experiencia docente, al iniciar la investigación manejábamos la hipótesis de que un alumno de secundaria, al completar la formación tradicional en la asignatura de Física, debía estar muy lejos de concebir la mecánica como un cuerpo de conocimientos mínimamente coherente, esperando que actuara como si ésta estuviera formada por parcelas intelectualmente desligadas. Sin embargo, no teníamos una opinión formada sobre la situación que se daría en el caso de los graduados universitarios.

El análisis de resultados se ha realizado desde tres vertientes distintas:

1. Evolución del «conocimiento físico» durante el bachilierato y la universidad.

2. Evolución de la estructura conceptual del alumno en mecánica.

3. Confianza en las respuestas.

\section{Evolución del «conocimiento físico»}

De aquí en adelante, denominaremos por «conocimiento físico» aquél que poseen los alumnos en formación y que viene caracterizado por ser una mezcla de saberes en los que proliferan ideas de «sentido común» acerca del funcionamiento de la naturaleza, donde los conceptos empleados no están bien delimitados y coexisten en aparente armonía con evidencias de que no resistirían el menor análisis, dirigido a lo evidente, etc. (Driver et al. 1989). También admitiría definición por contraposición con el conocimiento científico de la Física, sistemáticamente organizado, con conceptos que la intensa criba del tiempo ha permitido emplear sin contradicciones, con ámbitos que a medida que se conocen más encuentran unificación, etc.

En la tabla I se recogen los porcentajes de errores en cada uno de los ámbitos de estudio.

Tabla I

Errores por materias y niveles (\%).

\begin{tabular}{|l|c|c|c|c|c|}
\hline & ROZAMIENTO & GASES & FUERZA & CAIDA & ENERGIA \\
\hline PRIMERO & 92 & 60 & 96 & 100 & 92 \\
\hline SEGUNDO & 10 & 44 & 99 & 100 & 71 \\
\hline TERCERO & 9 & 58 & 95 & 99 & 63 \\
\hline COU & 7 & 47 & 80 & 91 & 58 \\
\hline GRAD.UNI. & 7 & 38 & 71 & 84 & 68 \\
\hline
\end{tabular}

Una ojeada a la tabla I muestra una caída generalizada en los porcentajes de error con el aumento de la formación científica. Esto es manifiesto si nos fijamos en los extremos ( $\left(1^{\circ}\right.$ de BUP y graduados $)$, sin embargo deja de ser tan significativo si lo hacemos a partir de $2^{\mathrm{g}}$ de BUP (inicio de la asignatura de Física y Química) y ponemos el techo en COU. En este caso, el análisis se limitaría a constatar que el conocimiento de los conceptos básicos de la mecánica no parece evolucionar durante el bachillerato, sobre todo teniendo en cuenta que desde $2^{\circ}$ de BUP a COU existen asignaturas específicas de Física y Química. El alumno continúa influido por su conocimiento previo extraescolar, manifestándose esa circunstancia con más o menos virulencia en función đel alejamiento o proximidad entre las interpretaciones física y previa del alumno (casos de fuerza y energía).

La universidad tampoco sirve para que sus graduados se vean libres de preconcepciones que tienen su origen en las primeras etapas de formación, hecho éste preocupante porque seguro que permitirá la retroalimentación del sistema, generando un círculo vicioso que perjudica a todos (Zalamea y Paris 1989).

En referencia a los distintos ámbitos de conocimiento analizados, citar por enésima vez la permanencia de la asociación fuerza-movimiento:

" (Una bola de billar que fue golpeada se mueve porque)... hay una fuerza que le permite moverse hacia delante ...» ( $3^{s}$ BUP). 
« (Un objeto lanzado hacia arriba sube porque) ...actúa la fuerza que permite que el objeto suba... la que nosotros hemos aplicadon ( $\left.3^{\circ} \mathrm{BUP}\right)$.

Los problemas para interpretar la caída de graves son muy grandes, incluso para los universitarios:

«(La maza caerá vertical porque) ...la fuerza de la gravedad actúa sobre la maza por ser más pesada, y provocaría el movimiento vertical» (Físico).

En cuanto al movimiento intrínseco de las partículas de los gases, cabe indicar que, aunque es aceptado, las explicaciones al respecto no reflejan un entendimiento del problema:

«(Las partículas đel aire en una nave espacial) ... estarían arriba $y$ en movimiento... si no hay gravedad, el aire estaría arriba pero seguiría moviéndose» (COU).

«...las partículas estarán en el fondo» (Químico).

Un hecho similar se plantea con la energía, concepto confuso que, además, suele asociarse con el de fuerza:

«...la energía (de la pelota) desaparece cuando llega al suelo por el choque"s ( $3^{\circ}$ de BUP).

«...si tu haces botar la pelota tarde o temprano se parará porque cesa la fuerzas ( $2^{\circ}$ de BUP).

«...la vagoneta pierde la fuerza» ( $2^{\circ}$ BUP).

En general, en las explicaciones dadas a las opciones escogidas, se plantean incongruencias con el empleo del lenguaje científico. La adopción de términos físicos en las argumentaciones dadas por los alumnos suele tener como trasfondo el solapamiento de una información científica con una concepción alternativa subyacente que aún permanece (Bell y Freyberg 1991).

Además de la presencia đe concepciones alternativas, el "conocimiento físico» viene caracterizado por la parcelación de los conceptos, los cuales llegan a ser manejados como compartimentos estancos. Como veremos a continuación, en la investigación realizada, esto ha quedado de manifiesto continuamente, pudiéndose usar como ejemplo de ello la independencia que muestra el conocimiento del concepto de fuerza o de la caída de graves, en relación con el nivel de conceptualización del rozamiento.

Como ya se ha indicado, el ítem relativo al rozamiento nos permitía clasifícar los encuestados según una tipología definida previamente, que diferencia entre:

\section{A. Desconocimiento del rozamiento.}

B. Limitación del rozamiento al deslizamiento de sólidos.

C. Ampliaciación del rozamiento a los fenómenos en medios fluidos
D. Caracterización plena del rozamiento, conocidos su origen, medios de actuación, etc.

Las tablas II y III muestran el porcentaje de errores de cada uno de estos grupos a los ítems relativos ai concepto de fuerza y a la caída de graves.

Tabla II

Errores en el concepto de fuerza para distintos niveles del concepto de rozaniento $(\%)$.

\begin{tabular}{|l|c|c|c|}
\hline & NIVELA & NTVEL B & NVEI C \\
\hline SEGUNDO & 100 & 100 & 99 \\
\hline YERCERO & 100 & 97 & 98 \\
\hline COU & 100 & 85 & 98 \\
\hline
\end{tabular}

Tabla III

Errores en caída para distintos niveles del concepto de rozamiento (\%).

\begin{tabular}{|l|c|c|c|}
\hline & NIVEL A & NIVEL B & NIVEL C . \\
\hline SEGUNDO & 100 & 100 & 100 \\
\hline TERCERO & 100 & 99 & 100 \\
\hline COU & 100 & 93 & 100 \\
\hline
\end{tabular}

Como puede apreciarse, no hay mejora en las respuestas aun cuando se avance, al menos aparentemente, en la conceptualización del rozamiento. Pudiera resultar evidente para muchos que la evolución en la comprensión de los fenómenos de fricción ayudara a comprender que en su ausencia los objetos caen igual con indepencia del peso, el movimiento de las partículas de un gas o la energía se conservan, etc. Sin embargo, los alumnos que a partir de $2^{Q}$ de BUP usan mayoritariamente el término de rozamiento (más del $90 \%$ ) no pueden emplearlo operativamente. Falta la coherencia característica del conocimiento científico, haciéndose necesario diferenciar, como hace Giordan (1988), entre nivel de formulación (conjunto de conocimientos necesarios para construir un enunciado, un estado de evolución en el desartollo psicogenético y una práctica social) y nivel lingüístico, que no es sino la expresión de un concepto a través de formulaciones totalmente gramaticales. Al menos en relación con el rozamiento se manifiesta un nivel lingüístico que no está en coherencia con el nivel de formulación.

La dificultad encontrada para aplicar el conocimiento que se tiene en el aprendizaje de nuevos conceptos puede tener implicaciones en la disposición temporal de los contenidos curriculares. Si la inducción de unos conceptos físicos a partir de otros no es algo automático sino que, por el contrario, se muestra ciertamente impedida, nos veremos en la necesidad de tratar conceptos anteriores cada vez que deseemos modificar o ampliar su campo de validez, relaciones, etc. Un currículo cíclico que retome repetidamente las temáticas puede resolver en parte el problema.

Profundizando en el tema de la fricción, podemos indicar que, si bien a partir de $2^{\circ}$ de BUP Ios alumnos 
reconocen la presencia de rozamiento, lo achacan mayoritariamente al deslizamiento de sólidos (Osborne 1991), aun cuando tal hecho no se dé. La fricción fluida no es tenida en cuenta por la mitad de los graduados y sólo una fracción anecdótica ( $2 \%$ ) de éstos manifiesta conocer el origen y mecanismo del rozamiento.

\section{Evolución de la estructura del «conocimiento físico»}

La concepción tradicional de la enseñanza de la ciencia como acumulación de saberes ha llevado a que normalmente se planteen unos contenidos enciclopédicos e inabordables que se exponen curso a curso. Sin embargo, como intuye cualquier profesor observador, y más adelante trataremos de demostrar, el alumno no los aprende de forma simultánea ni aun en el caso de una parcela restringida como la mecánica, que, además, rara vez se salva de ser planteada todos los años.

Según nuestros datos, la confrontación entre los esquemas previos del alumno y el conocimiento científico, provoca que el alumno se vaya «contaminando» poco a poco y de manera selectiva en algunas áreas de la asignatura, probablemente en función del arraigo de sus concepciones alternativas. La simultaneidad completa en el conocimiento significativo de los conceptos más usados en la mecánica no se produce ni en el caso de graduados en carreras científicas.

La adopción de algún sistema que permitiera representar la estructura del «conocimiento físico» de los encuestados fue desde el principio un problema a resolver. En el intento de hacer uso de un parámetro sencillo que fuera utilizable por la generalidad de un profesorado que se encuentra con dificultades para usar complicados análisis estadísticos, nos decantamos por el empleo de la probabilidad de conocer simultáneamente los hechos físicos analizados. Para ello necesitábamos manejar las respuestas individuales a cada uno de los ítems, de manera que fuera posible un análisis de coherencia en éstas. Por ejemplo, si en un momento dado nos planteamos el cálculo de la probabilidad de que los alumnos de $\mathrm{COU}$ conozcan simultánea y significaticamente los conceptos de energía y caída de graves, nos informaremos acerca del número de alumnos de ese curso que aciertan al mismo tiempo los ítems 5, 6 (caída), 7 y 8 (energía) para luego dividirlo por el número total de ellos. De igual manera se procedería con cualquier par de conceptos.

Este sencillo algoritmo permite elaborar tablas que recojan las probabilidades de conocer simultáneamente conceptos como los analizados en esta investigación en todos los niveles educativos (Tablas IV, V, VI, VII y VII). Éstas muestran las interconexiones que los alumnos establecen entre aquéllos, revelando de aiguna manera la estructura del «conocimiento físico». La aparición de un 0 en una casilla pone de relieve una ausencia completa de relación entre conceptos, así como la de un 1 manifiesta la certeza completa de que los alumnos de ese nivel los conozcan simultáneamente. En el primer caso, esa parcela de la estructura está por crear y en el segundo está completamente consolidada en ese nivel educativo. Una tabla formada mayoritariamente por ceros podría entenderse como producto de un grupo de alumnos con nula formación científica o bien con una formacion que, caracterizada por la parcelación, ha conseguido que los alumnos no sean capaces de conocer simultáneamente los conceptos físicos.

Una tabla formada únicamente por unos, hecho que no se da en los expertos oficiales de la materia, es esperable en la física que recogen los libros, aunque también esto habría quien lo discutiera.

Tabla IV

Probabilidad de conocimiento simuItáneo en primero de BUP.

\begin{tabular}{|l|c|c|c|c|c|}
\hline & ROZAMHENTO & $\begin{array}{c}\text { MOV. } \\
\text { GASES }\end{array}$ & FUERZA & CAIDA & ENERGIA \\
\hline ROZAMENTO & - & 0,04 & 0 & 0 & 0.04 \\
\hline MOV GAS & & - & 0 & 0 & 0.04 \\
\hline FUERZA & & & - & 0 & 0 \\
\hline CALDA & & & & - & 0 \\
\hline ENERGIA & & & & & $\vdots$ \\
\hline
\end{tabular}

Tabla V

Probabilidad de conocimiento simultáneo en segundo de BUP.

\begin{tabular}{|l|c|c|c|c|c|}
\hline & ROZAMIENTO & $\begin{array}{c}\text { MOV. } \\
\text { GASES }\end{array}$ & FUERZA & CAIDA & ENERGtA \\
\hline ROZAMIENTO & - & 0,55 & 0,01 & 0 & 0,29 \\
\hline MOV. GAS & & - & 0,01 & 0 & 0,20 \\
\hline FUERZA & & & - & 0 & 0 \\
\hline CAADA & & & & - & 0 \\
\hline ENERGIA & & & & & - \\
\hline
\end{tabular}

Tabla VI

Probabilidad de conocimiento simultáneo en tercero de BUP.

\begin{tabular}{|l|c|c|c|c|c|}
\hline & ROZAMIENTO & $\begin{array}{c}\text { MOV } \\
\text { GASES }\end{array}$ & FUERZA & CAIDA & ENERGIA \\
\hline ROZAMIENTO & - & 0,38 & 0,05 & 0,01 & 0,34 \\
\hline MOV. GAS & & - & 0 & 0 & 0,19 \\
\hline FUERZA & & & & 0 & 0,01 \\
\hline CAIDA & & & & & 0 \\
\hline ENERGIA & & & & & - \\
\hline
\end{tabular}

Tabla VII

Probabilidad de conocimiento simuitáneo en COU.

\begin{tabular}{|l|c|c|c|c|c|}
\hline & ROZAMENTO & $\begin{array}{c}\text { MOV } \\
\text { GASES }\end{array}$ & FUERZA & CAIDA & ENERGtA \\
\hline ROZAMIENTO & - & 0.53 & 0,20 & 0,09 & 0,42 \\
\hline MOV GAS & & - & 0.16 & 0,04 & 0,20 \\
\hline FUERZA & & & - & 0,04 & 0,07 \\
\hline CAIDA & & & & - & 0,04 \\
\hline ENERGIA & & & & & - \\
\hline
\end{tabular}

ENSEÑANZA DE LAS CIENCIAS, 1993, 11 (2) 
Tabla VIII

Probabilidad de conocimiento simultáneo en graduados en ciencias.

\begin{tabular}{|l|c|c|c|c|c|}
\hline & ROZAMIENTO & $\begin{array}{c}\text { MOV. } \\
\text { GASES }\end{array}$ & FUERZA & CALAA & ENERGIA \\
\hline ROZAMIENTO & - & 0,58 & 0,26 & 0,13 & 0,32 \\
\hline MOV. GAS & & - & 0.23 & 0,10 & 0,29 \\
\hline FUERZA & & & - & 0,13 & 0,10 \\
\hline CADA & & & & - & 0,03 \\
\hline ENEROIA & & & & & - \\
\hline
\end{tabular}

El análisis de las tablas IV a VIII tiene múltiples aspectos:

- Desde $1^{8}$ de BUP hasta graduados universitarios, el número de casillas con valores nulos evoluciona del siguiente modo: $7,5,4,0$ y 0 . Es decir, que frente a 7 relaciones no establecidas en $1^{9}$ de BUP, en alumnos de COU. y graduados no se echa en falta ninguna, manifestândose una evolución de la estructura del «conocimiento científico" que se dirige hacia el aumento en la complejidad. Tơo ello resulta aún más revelador cuando comparamos estos datos con la tabla I. En ella, que recogía los porcentajes de error en cada ámbito de estudio, indicábamos que no se manifestaban las diferencias entre los niveles educativos tan claramente como cabría esperar; ahora, sin embargo, la «calidad» de unas y otras respuestas queda patente. Mientras que, en los aspectos estudiados, entre alumnos de 2 de BUP y graduados sólo hay una diferencia media del $9 \%$ en los porcentajes de error, los últimos manifiestan una trama de relaciones que no poseen los primeros, mostrando una coherencia que no se tiene en las etapas educativas más tempranas.

- Si nos fijamos en casillas concretas, en general puede verse que al aumentar la formación crece la probabilidad de conocer simultáneamente los conceptos, revelando un mayor afianzamiento en la red de conocimientos. Por ejemplo, en la figura I se recogen los datos de las parejas fuerza-rozamiento y fuerza-energía.

Figura I

Conocimiento simultáneo.

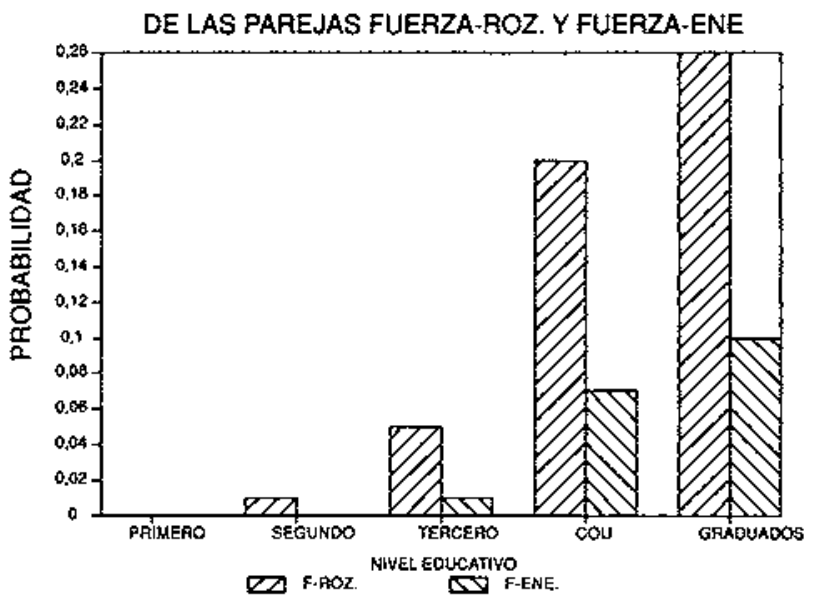

- Se puede constatar la dificultad que tienen los alumnos para crear un cuerpo coherente de conocimientos con todas las informaciones que en tal sentido le liegan. Al igual que se aprecia una evolución a medida que avanza la formación, es claro también que ni al final del bachillerato ni de la formación universitaria, se ha conseguido estructurar completamente el conocimiento científico en la mecánica. Puede afirmarse que, con independencia del nivel de formación, la física como tal no está en la cabeza de los alumnos. Faltan conexiones básicas que con seguridad le restan potencia explicativa, haciendo necesario el uso de argumentaciones paralelas o de "sentido común» para encontrar soluciones que de otro modo resultarían evidentes.

Estos hechos debexían tener consecuencias sobre el proceso de enseñanza/aprendizaje de las ciencias. Por una parte, la figura del profesor «sabelotodo» queda en evidencia, entrando él mismo a formar parte de un proceso en el que también se ven inmersos sus alumnos. Las connotaciones sociológicas que debe tener este hecho son muy importantes, ya que permiten entender al alumno como un constructor de su propio conocimiento y no como un depósito de errores reconocibles desde la ciencia acabada de su profesor. Ello tiene que influir en la modificación del clima del aula, cuestión básica para que puedan consolidarse opciones curriculares dirigidas a enfocar la enseñanza de la ciencia como un proceso de investigación (Gil et al. 1991). Por otra parte, no hay que olvidar el desarrolio cognitivo de los estudiantes. EI fracaso de nuestro programa educativo puede estar en relación con su inadaptación tanto a las posibilidades cognitivas de aquéllos (Shayer y Adey 1986), como a la trama de relaciones conceptuales que en un momento dado puedan establecer los alumnos.

\section{Confianza en las respuestas}

En la descripción de la encuesta empleada en la investigación, se ha citado que se preguntaba a los alumnos sobre el nivel de confianza que tenían en sus respuestas. Ello se hacía con una calificación de 0 a 10 que recogía todo el abanico de posibilidades entre confianza nula y absoluta.

Nos ha llamado la atención el hecho de que los encuestados, en general, mostraban una notable confianza en sus respuestas, acertadas o no, siendo escasas las ocasiones en que, por término medio en un curso, eran inferiores a 5 puntos en un ítem. En la tabla IX se recogen los valores medios de confianza en cada temática estudiada.

Tabla IX

Confianza en las respuestas.

\begin{tabular}{|l|c|c|c|c|c|}
\hline & ROZAMIENTO & $\begin{array}{c}\text { MOV. } \\
\text { GASES }\end{array}$ & FUERZA & CAIDA & ENERGIA \\
\hline CONFIANZA & 6,7 & 5,2 & 7,8 & 7,1 & 6,2 \\
\hline DESV. TIPIC. & 2,6 & 2,8 & 2,4 & 2,6 & 2,8 \\
\hline
\end{tabular}


Figura 2

Acierto y confianza.

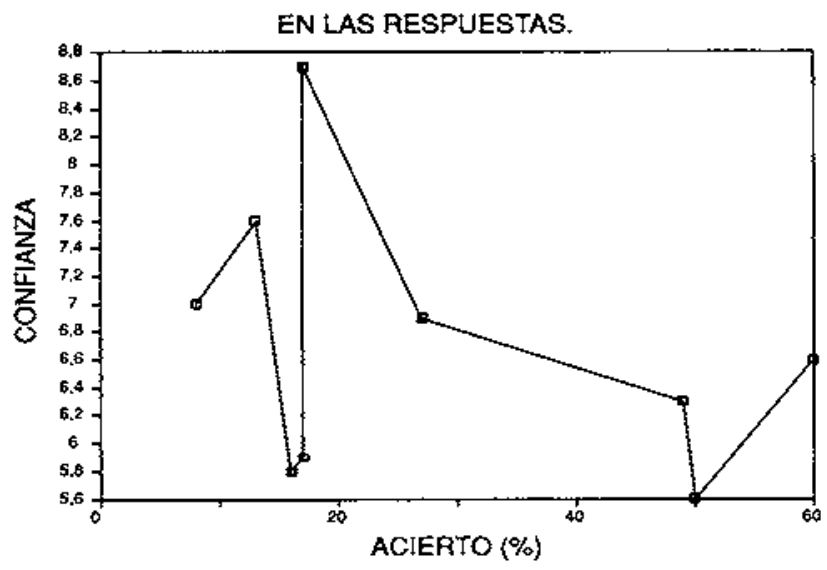

Si nos centramos en las opciones correctas de cada f́tem, resulta curioso comprobar que, con independencia del porcentaje de alumnos que opten por ella, son las que ofrecen más confianza en el $70 \%$ de los ítems; dato significativo si tenemos en cuenta que se trata de una opción entre, al menos, cuatro posibles.

Como ha podido apreciarse, la menor confianza se asocia al comportamiento de la materia en estado gaseoso. La mayor confianza a la naturaleza de las fuerzas.

La circunstancia de que el concepto de fuerza fuera mal conocido por los estudiantes y, sin embargo, Ias respuestas relacionadas con él mostraran gran confianza, hizo que nos planteáramos el estudio de la relación que guardan ambos aspectos. Para ello analizamos la correlación existente entre los valores medios de aciertos y confianza en cada ítem, encontrando que el coeficiente

\section{REFERENCIAS BIBLIOGRÁFICAS}

AUSUBEL, D.P., NOVACK, J.D. y HANESIAN, H., I983. Psicologla Educativa. (Trillas: México).

BELL, B. y FREYBERG, P., 1991. El lenguaje en la clase de ciencias, en Osborne, R., Freyberg, P., El aprendizaje de las ciencias. (Narcea: Madrid).

DRIVER, R., GUESNE, E. y TIBERGHIEN, A., 1989. Ideas científicas en la infancia y la adolescencia. (MEC/Morata: Madrid).

DRIVER, R., 1988. Un enfoque constructivista para el desarrollo del curriculo de ciencias, Enseñanza de las Ciencias, 6(2), pp. 109-120.

GAGLIARDI, R, 1988. Cómoutilizar la Historia de las ciencias en la enseñanza de las ciencias, Enseñanza de las Ciencias, 6 (3), pp. 291-296.

GIL, D., CARRASCOSA, J., FURIÓ, C. y MARTINEZ, J., 1991. Laenseñanza de las ciencias en la educación secundaria. (ICE Universitat de Barcelona / Horsori: Barcelona).

GIORDAN, A. y de VECCHI, G., 1988. Los origenes del saber. (Díada: Sevilia). de correlación es de $-0,4$ (Fig. 2). La independencia de variables que sugería este análisis, la confirmamos repitiendo el proceso en cada curso. Efectivamente, no existe relación entre la confianza que depositan los alumnos en una respuesta y el acierto que se desprende de ella.

\section{CONCLUSIONES}

Resumiendo lo más significativo del trabajo realizado, podemos citar lo siguiente:

- El conocimiento de la mecánica evoluciona con Ientitud durante el bachillerato y aun en la universidad, denotándose parcelas ya clásicas donde las ideas previas permanecen hasta etapas tan postreras como las de graduado universitario.

- La estructura del "conocimiento físico» puede esbozarse mediante análisis de ideas previas. La complejidad de aquél aumenta con la formación científica, pưđiendo ser empleada para diferenciar a alumnos de diferentes etapas educativas. Hay relaciones que se establecen tardiamente, lo cual debe guardar relación con la persistencia de las ideas previas y la ineficacia de la enseñanza recibida para modificarlas y, consecuentemente, construir un cuerpo coherente de conocimientos. La parcelación es una característica fundamental del «conocimiento físico» de Ios alumnos en su etapa formativa. Aun después de graduarse están muy lejos de poner orden en el cúmulo de informaciones recibidas. La inferencia de conceptos físicos a partir de otros no se produce con facilidad.

- La confianza mostrada por los alumnos en sus respuestas a cuestiones básicas de la mecánica no guarda relación con el acierto en las mismas.

GIORDAN, A., 1989. Representaciones sobre la utilizacion didáctica de las representaciones, Enseñanza de las Ciencias, 7 (1), pp. 53-62.

HIERREZUELO, J. Y MONTERO, A., 1989. La ciencia de los alumnos. (Laia/MEC: Barcelona).

OSBORNE, R., 1991. Construir a partir de las ideas intituitivas de los alumnos, en Osborne, R. y Freyberg, P., Elaprendizaje de las ciencias. (Narcea: Madrid).

POZO, J.I., 1987. Aprendizaje de la ciencia y pensamiento causal. (Visor: Madrid).

POZO, I., SANZ, A., GOMEZ, M. y LIMÓN, M., 1991. Las ideas de los alumnos sobre ciencia: una interpretacion desde la psicología cognitiva, Enseñanza de las Ciencias, 9 (1), pp. 83-94.

SHAYER, M. y ADEY, Ph., 1986. La ciencia de enseñar ciencias. (Narcea, Madrid).

ZALAMEA, E. y PARIS, R., 1989. ¿Saben los maestros ia física que enseñan?, Enseñanza de las Ciencias, 7 (3), pp. $251-256$ 


\section{ANEXO I}

Un vehíctulo a motor va por una carretera a una velocidad $v$.

Dibuja Ias fuerzas que actúan sobre el vehículo explicando su origen.

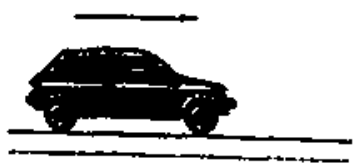

$\mathrm{V}=90 \mathrm{~km} / \mathrm{h}$

Fuerza hacia la derecha:

Explica su origen:

Fuerza hacia la izquierda:

Explica su origen:

Indica el nivel de seguridad

en la respuesta $(0=$ nula, $10=$ completa $)$

Explica brevemente el sentido de tu respuesta:

EI Voyager I y II son naves que fueron lanzadas en 1977 y se espera que sigan moviéndose durante mucho tiempo. Teniendo en cuenta que el aire está formado por partículas en movimiento, si dentro de miles o millones de años fueran encontradas, ¿cómo estarían distribuidas las partículas de aire en el interior de las naves?

a)

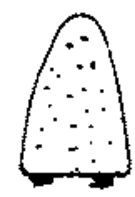

Igual.

Se moverían igual de rápidas. b)

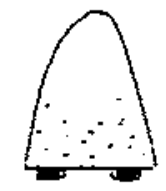

Habría más en la parte baja. Irían más lentas. c)

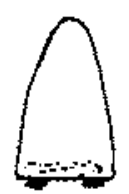

Todas en el fondo. Se habrian parado. d)

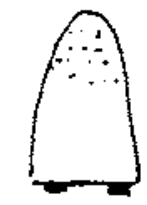

Todas arriba.

Se habrían parado.

Indica el nivel de seguridad

en la respuesta $(0=$ nula, $10=$ completa $)$.

Explica brevemente el sentido de tu respuesta: 
La figura representa una bola de billar que ha sido golpeada por el taco y avanza por la superficie horizontal de la mesa. Mientras se está moviendo, las fuerzas horizontales que le están aplicadas son:

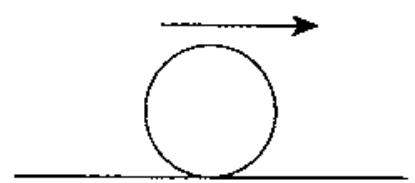

a)

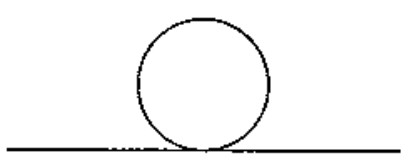

Ninguna

c)

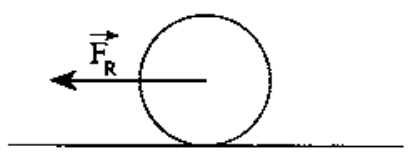

e) Otra respuesta b)

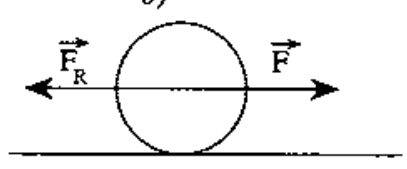

d)

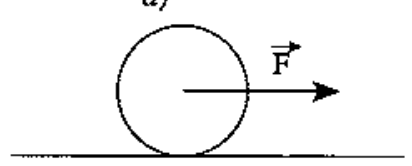

f) No lo sé

Indica el nivel de seguridad

en la respuesta $(0=$ nula, $10=$ completa $)$

Explica brevemente el sentido de tu respuesta:

La figura representa un objeto que ha sido lanzado verticalmente hacia arriba.

Mientras sube, las fuerzas que le están aplicadas son:

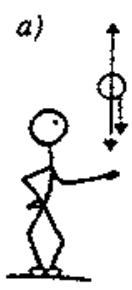

e) Otra respuesta

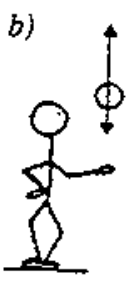

c)

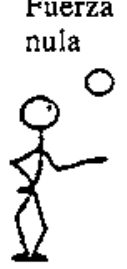

f) No lo sé

Indica el nivel de seguridad

en la respuesta $(0=$ nula, $10=$ completa $)$

Explica brevemente el sentido de tu respuesta: 
La maza de un tambor que inicialmente se encontraba en posición horizontal, se deja caer. ¿Cómo se movería durante la caída?

a)

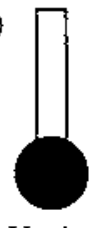

Vertical

c)

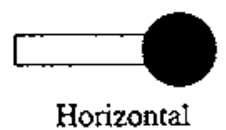

e) Otra respuesta b)

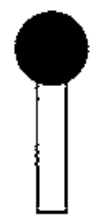

Vertical

d)

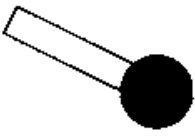

Inclinado

Indica el nivel de seguridad

en la respuesta $(0=$ nula, $10=$ completa $)$

Explica brevemente el sentido de tu respuesta:

f) No lo sé

Dos bolas del mismo tamaño, una con doble masa que la otra, son lanzadas verticaimente hacia arriba con idéntica velocidad inicial. ¿Que relación existe entre las alturas máximas que alcanzan?

a) Son iguales.

b) La más pesada llega más alta porque tiene más inercia.

c) La menos pesada Ilega más arriba.

d) La menos pesada alcanza el doble de altura.

e) No lo sé.

f) Otra respuesta.

Indica el nivel de seguridad

en la respuesta $(0=$ nula, $10=$ completa $)$

Explica brevemente el sentido de tu respuesta: 
Estamos acostumbrados a comprobar que, cuando dejamos caer libremente una pelota desde cierfa altura después de rebotar, no vuelve a alcanzar el punto de partida sino que queda algo más abajo. Esto supone que:

a) La energía de la pelota ha aumentado.

b) La energía de la pelota ha disminuido.

c) La energía de la pelota continúa igual.

d) La energía no tiene ningún poder explicativo para este fenómeno.

e) No lo sé.

f) Otra respuesta.

Indica el nivel de seguridad

en la respuesta $(0=$ nula, $10=$ completa $)$

Explica brevemente el sentido de tu respuesta:

Vagonetas sin motor son normalmente empleadas para el traslado de materiales por el interior de las galerías de las minas. Si, por accidente, una de ellas que se encontraba detenida en el punto $\mathrm{P}$ (ver figura) se pone en movimiento, podemos afirmar que tiene energía sufictente para hacer peligrar a los mineros que están en los tramos:

a) A-B

b) $\mathrm{A}-\mathrm{B}$ y $\mathrm{B}-\mathrm{C}$

c) $\mathrm{A}-\mathrm{B}, \mathrm{B}-\mathrm{C}$ y $\mathrm{C}-\mathrm{D}$

d) A-B, B-C, C-D y D-E

e) No lo sé

f) Otra respuesta

Indica el nivel de seguridad

en la respuesta $(0=$ nula, $10=$ completa $)$

Explica brevemente el sentido de tu respuesta: 